\title{
Finite-rank perturbations of positive operators and isometries
}

\author{
by \\ Man-Duen Choi (Toronto) and Pei Yuan Wu (Hsinchu)
}

\begin{abstract}
We completely characterize the ranks of $A-B$ and $A^{1 / 2}-B^{1 / 2}$ for operators $A$ and $B$ on a Hilbert space satisfying $A \geq B \geq 0$. Namely, let $l$ and $m$ be nonnegative integers or infinity. Then $l=\operatorname{rank}(A-B)$ and $m=\operatorname{rank}\left(A^{1 / 2}-B^{1 / 2}\right)$ for some operators $A$ and $B$ with $A \geq B \geq 0$ on a Hilbert space of dimension $n(1 \leq n \leq \infty)$ if and only if $l=m=0$ or $0<l \leq m \leq n$. In particular, this answers in the negative the question posed by C. Benhida whether for positive operators $A$ and $B$ the finiteness of $\operatorname{rank}(A-B)$ implies that of $\operatorname{rank}\left(A^{1 / 2}-B^{1 / 2}\right)$.

For two isometries, we give necessary and sufficient conditions in order that they be finite-rank perturbations of each other. One such condition says that, for isometries $A$ and $B, A-B$ has finite rank if and only if $A=(I+F) B$ for some unitary operator $I+F$ with finite-rank $F$. Another condition is in terms of the parts in the Wold-Lebesgue decompositions of the nonunitary isometries $A$ and $B$.
\end{abstract}

A bounded linear operator $A$ on a complex separable Hilbert space $H$ is said to be positive, denoted by $A \geq 0$, if $\langle A x, x\rangle \geq 0$ for all vectors $x$ in $H$, where $\langle\cdot, \cdot\rangle$ denotes the inner product in $H$. If $A$ is positive, then $A^{1 / 2}$ denotes the (unique) positive square root of $A$. In a recent paper by C. Benhida [1], it was asked whether for two positive operators $A$ and $B$, the condition $\operatorname{rank}(A-B)<\infty$ would imply $\operatorname{rank}\left(A^{1 / 2}-B^{1 / 2}\right)<\infty$. In Section 1 below, we completely characterize the ranks of $A-B$ and $A^{1 / 2}-B^{1 / 2}$ for operators $A$ and $B$ satisfying $A \geq B \geq 0$. We show, in particular, that the answer to Benhida's question is "No". On the other hand, if $A$ and $B$ are commuting positive operators, then the rank of $A-B$ equals that of $A^{1 / 2}-B^{1 / 2}$.

We next consider, in Section 2, two isometries $V_{1}$ and $V_{2}$ and give two different necessary and sufficient conditions in order that they be finiterank perturbations of each other. One condition converts the finite-rank perturbation of $V_{1}$ and $V_{2}$ into a multiplicative unitary relation between

2000 Mathematics Subject Classification: Primary 47A55, 47B15, 47B20.

Key words and phrases: finite-rank perturbation, positive operator, isometry, WoldLebesgue decomposition. 
them. Another condition is in terms of the parts in the Wold-Lebesgue decompositions of the nonunitary $V_{1}$ and $V_{2}$.

For any operator $A$, we use ker $A$ and range $A$ to denote the kernel and range of $A$, respectively. The $\operatorname{rank}$ of $A, \operatorname{rank} A$, is the dimension of $\overline{\operatorname{range} A}$. A (closed) subspace $K$ of $H$ is said to reduce the operator $A$ on $H$ if $A K$ and $A^{*} K$ are contained in $K$, in which case $A$ can be decomposed as $A_{1} \oplus A_{2}$ on $H=K \oplus K^{\perp}$.

1. Positive operators. The main result of this section is the following theorem giving a characterization of the pairs of integers which are the ranks of $A-B$ and $A^{1 / 2}-B^{1 / 2}$ for operators $A$ and $B$ satisfying $A \geq B \geq 0$.

THEOREM 1.1. Let $l$ and $m$ be nonnegative integers or infinity. Then $l=\operatorname{rank}(A-B)$ and $m=\operatorname{rank}\left(A^{1 / 2}-B^{1 / 2}\right)$ for some operators $A$ and $B$ with $A \geq B \geq 0$ on a Hilbert space of dimension $n(1 \leq n \leq \infty)$ if and only if $l=m=0$ or $0<l \leq m \leq n$.

The necessity of the condition is proved in the next lemma.

LEMMA 1.2.

(a) If $A$ and $B$ are positive operators on the same Hilbert space $H$, then $\operatorname{rank}(A-B) \leq 2 \operatorname{rank}\left(A^{1 / 2}-B^{1 / 2}\right)$.

(b) If $A$ and $B$ on $H$ satisfy $A \geq B \geq 0$, then we have $\operatorname{rank}(A-B) \leq$ $\operatorname{rank}\left(A^{1 / 2}-B^{1 / 2}\right)$ and $\operatorname{ker}\left(A^{1 / 2}-B^{1 / 2}\right)$ is a common reducing subspace of $A$ and $B$.

Proof. The assertion in (a) follows from the equality

$$
A-B=A^{1 / 2}\left(A^{1 / 2}-B^{1 / 2}\right)+\left(A^{1 / 2}-B^{1 / 2}\right) B^{1 / 2} .
$$

To prove (b), let $F=A^{1 / 2}-B^{1 / 2}$ and $K=\operatorname{ker} F$. Assume that $F$ and $B^{1 / 2}$ are represented as

$$
F=F_{1} \oplus 0, \quad B^{1 / 2}=\left[\begin{array}{cc}
B_{1} & B_{2} \\
B_{2}^{*} & B_{3}
\end{array}\right]
$$

on $H=K^{\perp} \oplus K$. Then

$$
\begin{aligned}
A-B & =\left(B^{1 / 2}+F\right)^{2}-B=B^{1 / 2} F+F B^{1 / 2}+F^{2} \\
& =\left[\begin{array}{cc}
B_{1} F_{1}+F_{1} B_{1}+F_{1}^{2} & F_{1} B_{2} \\
B_{2}^{*} F_{1} & 0
\end{array}\right] \geq 0 .
\end{aligned}
$$

This implies that $F_{1} B_{2}=0$. Since $F_{1}$ is one-to-one, we obtain $B_{2}=0$. Thus $A-B=\left(B_{1} F_{1}+F_{1} B_{1}+F_{1}^{2}\right) \oplus 0$, from which we derive that $\operatorname{ker}(A-B) \supseteq$ $\operatorname{ker}\left(A^{1 / 2}-B^{1 / 2}\right)$. Hence

$$
\overline{\operatorname{range}(A-B)} \subseteq \overline{\operatorname{range}\left(A^{1 / 2}-B^{1 / 2}\right)}
$$


and so $\operatorname{rank}(A-B) \leq \operatorname{rank}\left(A^{1 / 2}-B^{1 / 2}\right)$. The assertion on $\operatorname{ker}\left(A^{1 / 2}-B^{1 / 2}\right)$ follows from the arguments above.

To prove the sufficiency of the condition in Theorem 1.1, we need the following lemma. This should be known to experts. We include the proof for completeness.

LEMMA 1.3. If $A=\left[a_{i j}\right]_{i, j=1}^{n}$ is a matrix with $a_{i j} \neq 0$ for all $i$ and $j$, and $B$ is a diagonal matrix $\operatorname{diag}\left(b_{1}, \ldots, b_{n}\right)$ with distinct $b_{j}$ 's, then the only common reducing subspaces of $A$ and $B$ are the trivial ones $\{0\}$ and $\mathbb{C}^{n}$. The analogous assertion (with $n$ replaced by infinity) holds for infinite matrices $A$ and $B$ on $l^{2}$.

Proof. Let $M$ be a common reducing subspace of $A$ and $B$, and let $P_{M}$ be the (orthogonal) projection from $\mathbb{C}^{n}$ onto $M$. Since $P_{M}$ commutes with $B$ and the $b_{j}$ 's are distinct, we derive that $P_{M}=\operatorname{diag}\left(p_{1}, \ldots, p_{n}\right)$ with $p_{j}=0$ or 1 for each $j$. On the other hand, since $P_{M}$ also commutes with $A$ and the entries of $A$ are all nonzero, we conclude that either $p_{j}=0$ for all $j$ or $p_{j}=1$ for all $j$. Hence $M$ can only be $\{0\}$ or $\mathbb{C}^{n}$ as asserted.

Note that in the preceding lemma the requirement on the entries of $A$ can be considerably weakened. However, for our purposes the present form suffices.

Proof of Theorem 1.1. We first assume that $0<l \leq m=n<\infty$. Let $B=\operatorname{diag}\left(b_{1}, \ldots, b_{m}\right)$ be a diagonal matrix with positive and distinct $b_{j}$ 's, let $x_{1}, \ldots, x_{l}$ be $l$ linearly independent vectors in $\mathbb{C}^{m}$ whose components are all positive, let $C=\sum_{j=1}^{l} x_{j} x_{j}^{*}$, and let $A=B+C$. Then $A \geq B \geq 0, \operatorname{rank} C=l$ and the entries of $A$ are all positive. By Lemma 1.3, the only common reducing subspaces of $A$ and $B$ are $\{0\}$ and $\mathbb{C}^{m}$. Since $\operatorname{ker}\left(A^{1 / 2}-B^{1 / 2}\right)$ is a common reducing subspace of $A$ and $B$ by Lemma $1.2(\mathrm{~b})$, we conclude that $\operatorname{ker}\left(A^{1 / 2}-B^{1 / 2}\right)=\{0\}$ or $\mathbb{C}^{m}$. The latter is impossible since $A$ and $B$ are not equal. Hence $\operatorname{ker}\left(A^{1 / 2}-B^{1 / 2}\right)=\{0\}$ and thus $\operatorname{rank}\left(A^{1 / 2}-B^{1 / 2}\right)=m$. For the more general case that $0<l \leq m \leq n<\infty$, let $A$ and $B$ be the $m$-by-m matrices as above. Then $A \oplus 0_{n-m}$ and $B \oplus 0_{n-m}$ meet our requirements. Analogous constructions of $A$ and $B$ work for $n=\infty$. This completes the proof.

We conclude this section with two related facts. Firstly, if $A$ and $B$ are commuting positive operators, then $\operatorname{rank}(A-B)=\operatorname{rank}\left(A^{1 / 2}-B^{1 / 2}\right)$. This can be deduced from the spectral theory of the normal operator $A+i B$. In the next proposition, we prove this from some easily derived facts.

Proposition 1.4. If $A$ and $B$ are commuting positive operators, then

$$
\overline{\operatorname{range}(A-B)}=\overline{\operatorname{range}\left(A^{1 / 2}-B^{1 / 2}\right)} \text {. }
$$


Proof. The commuting of $A$ and $B$ implies that of $A^{1 / 2}$ and $B^{1 / 2}$. Hence $A-B=\left(A^{1 / 2}+B^{1 / 2}\right)\left(A^{1 / 2}-B^{1 / 2}\right)$. From this, we deduce the inclusion $\operatorname{ker}\left(A^{1 / 2}-B^{1 / 2}\right) \subseteq \operatorname{ker}(A-B)$. On the other hand, from

$$
0 \leq\left(A^{1 / 2}-B^{1 / 2}\right)^{4}=(A-B)^{2}-4 A^{1 / 2} B^{1 / 2}\left(A^{1 / 2}-B^{1 / 2}\right)^{2} \leq(A-B)^{2},
$$

we obtain $\operatorname{ker}(A-B) \subseteq \operatorname{ker}\left(A^{1 / 2}-B^{1 / 2}\right)$. Thus $\operatorname{ker}(A-B)=\operatorname{ker}\left(A^{1 / 2}-B^{1 / 2}\right)$ and our assertion follows.

Secondly, it is known that the compactness of $A-B$ for positive operators $A$ and $B$ implies that of $A^{1 / 2}-B^{1 / 2}$. Not being able to find a precise reference, we provide a proof below.

Proposition 1.5. Let $A$ and $B$ be positive operators on the same space. If $A-B$ is compact, then so is $A^{1 / 2}-B^{1 / 2}$.

Proof. It is easily seen that if $A-B$ is compact, then so is $p(A)-p(B)$ for any polynomial $p$. Let $p_{n}, n=1,2, \ldots$, be a sequence of polynomials which converges uniformly to the square-root function $f(t)=\sqrt{t}$ on $\sigma(A) \cup \sigma(B)$. Then $p_{n}(A)$ and $p_{n}(B)$ converge in norm to $A^{1 / 2}$ and $B^{1 / 2}$, respectively. Hence $A^{1 / 2}-B^{1 / 2}$, being the norm limit of the compact operators $p_{n}(A)-$ $p_{n}(B)$, is also compact.

2. Isometries. An operator $A$ is an isometry if $\|A x\|=\|x\|$ for any vector $x$. In this section, we obtain two different kinds of necessary and sufficient conditions for two isometries to be finite-rank perturbations of each other. The first of these is one which converts the additive finite-rank perturbation into a "left" multiplicative unitary perturbation.

TheOREM 2.1. Let $V_{1}, V_{2}$ be isometries on a separable Hilbert space $H$. Then $\operatorname{rank}\left(V_{1}-V_{2}\right)<\infty$ if and only if there is a unitary operator $U$ of the form $I+F$ with $\operatorname{rank} F<\infty$ such that $V_{1}=U V_{2}$. Moreover, in this case, $F$ can be chosen with $\operatorname{rank} F \leq 2 \operatorname{rank}\left(V_{1}-V_{2}\right)$.

Proof. Assume that $K \equiv \operatorname{range}\left(V_{1}^{*}-V_{2}^{*}\right)$ is finite-dimensional. Then so is $L \equiv V_{1}(K)+V_{2}(K)$. Obviously, we have $V_{j}(K) \subseteq L$ for $j=1,2$. On the other hand, since for any $x$ in $K^{\perp}$ and $y$ in $K$ the equalities

$$
\left\langle V_{j} x, V_{j} y\right\rangle=\left\langle x, V_{j}^{*} V_{j} y\right\rangle=\langle x, y\rangle=0
$$

hold, we obtain $V_{j}\left(K^{\perp}\right) \subseteq\left(V_{j} K\right)^{\perp}$. Together with the fact that $V_{1}=V_{2}$ on $\operatorname{ker}\left(V_{1}-V_{2}\right)=K^{\perp}$, this yields $V_{j}\left(K^{\perp}\right) \subseteq\left(V_{1} K\right)^{\perp} \cap\left(V_{2} K\right)^{\perp}=L^{\perp}, j=1,2$. Consider the 2-by-2 operator matrix representation

$$
V_{j}=\left[\begin{array}{cc}
W_{j} & 0 \\
0 & R
\end{array}\right]
$$

of $V_{j}$ from $H=K \oplus K^{\perp}$ to $H=L \oplus L^{\perp}$, where $W_{j}: K \rightarrow L$ and $R$ : $K^{\perp} \rightarrow L^{\perp}$ are isometries. Since $W_{2} W_{1}^{*} \mid W_{1} K$ is an isometry mapping $W_{1} K$ 
onto $W_{2} K$, it can be extended to a unitary operator $U_{0}$ on the (finitedimensional) space $L$. Let $U=U_{0} \oplus I$ on $H=L \oplus L^{\perp}$. Then $U$ is unitary with $\operatorname{rank}(U-I) \leq 2 \operatorname{rank}\left(V_{1}-V_{2}\right)$ and satisfies

$$
\begin{aligned}
U V_{1} & =\left[\begin{array}{cc}
U_{0} & 0 \\
0 & I
\end{array}\right]\left[\begin{array}{cc}
W_{1} & 0 \\
0 & R
\end{array}\right]=\left[\begin{array}{cc}
U_{0} W_{1} & 0 \\
0 & R
\end{array}\right] \\
& =\left[\begin{array}{cc}
W_{2} W_{1}^{*} W_{1} & 0 \\
0 & R
\end{array}\right]=\left[\begin{array}{cc}
W_{2} & 0 \\
0 & R
\end{array}\right]=V_{2},
\end{aligned}
$$

completing the proof.

It is easier to prove the corresponding "right" multiplicative unitary perturbation for isometries.

Proposition 2.2. Let $V_{1}$ and $V_{2}$ be isometries on a common Hilbert space. Then there exists a unitary operator $U$ of the form $I+F$ with finiterank $F$ such that $V_{1}=V_{2} U$ if and only if range $V_{1}=\operatorname{range} V_{2}$ and $\operatorname{rank}\left(V_{1}-V_{2}\right)$ $<\infty$. Moreover, in this case, $\operatorname{rank} F$ is equal to $\operatorname{rank}\left(V_{1}-V_{2}\right)$.

Proof. If $V_{1}=V_{2} U$ as above, then obviously range $V_{1}=$ range $V_{2}$ and also

$$
\operatorname{rank}\left(V_{1}-V_{2}\right)=\operatorname{rank} V_{2}(U-I)=\operatorname{rank} V_{2} F=\operatorname{rank} F<\infty .
$$

Conversely, if range $V_{1}=$ range $V_{2}$, then Douglas's factorization theorem [5, Problem 59] implies that $V_{1}=V_{2} U$ for some invertible operator $U$. Since both $V_{1}$ and $V_{2}$ are isometries, so is $U$. Hence $U$ is unitary. Moreover, $\operatorname{rank}(U-I)=\operatorname{rank}\left(V_{1}-V_{2}\right)<\infty$ follows as above. This completes the proof.

We now come to the second condition for the finite-rank perturbations of nonunitary isometries.

THEOREM 2.3. Let $V_{1}$ and $V_{2}$ be nonunitary isometries on a separable Hilbert space. Then $\operatorname{rank}\left(V_{1}-U^{*} V_{2} U\right)<\infty$ for some unitary $U$ if and only if $V_{j}$ is unitarily equivalent to $U_{j} \oplus W, j=1,2$, where $U_{1}$ and $U_{2}$ are singular unitary operators with finite multiplicity and $W$ is a nonunitary isometry.

Recall that the multiplicity $\mu(A)$ of an operator $A$ on $H$ is the minimum cardinality of a subset $\left\{x_{\lambda}\right\}_{\lambda \in \Lambda}$ of $H$ for which the closed linear span of the vectors $A^{n} x_{\lambda}, n \geq 0$ and $\lambda \in \Lambda$, equals $H$. The operator $A$ is said to be cyclic if $\mu(A)=1$. By the spectral theorem, a normal operator has finite multiplicity if and only if it is the direct sum of finitely many cyclic operators (cf. [4, Section IX.10]).

For the proof of Theorem 2.3, we need the Wold-Lebesgue decomposition of isometries. This says that every isometry $V$ can be uniquely decomposed as the direct sum of a singular unitary operator $U_{s}$, an absolutely continuous 
unitary operator $U_{a}$ and a unilateral shift $S^{(n)}: V=U_{s} \oplus U_{a} \oplus S^{(n)}$. Here $S^{(n)}$ denotes the direct sum of $n$ copies $(0 \leq n \leq \infty)$ of the simple unilateral shift $S$. The proof for the sufficiency of Theorem 2.3 is based on the following lemma.

Lemma 2.4. Let $S^{(n)}(1 \leq n \leq \infty)$ be the direct sum of $n$ copies of the simple unilateral shift. Then an isometry is a rank-one perturbation of $S^{(n)}$ if and only if it is unitarily equivalent to either $S^{(n)}$ or $U \oplus S^{(n)}$, where $U$ is a cyclic singular unitary operator.

This is proved in [6, Theorem 2 and Proposition 2].

We are now ready to prove Theorem 2.3.

Proof of Theorem 2.3. For $j=1,2$, let $V_{j}=U_{j s} \oplus U_{j a} \oplus S^{\left(n_{j}\right)}$ be the Wold-Lebesgue decomposition of $V_{j}$ as above with $1 \leq n_{j} \leq \infty$.

To prove one direction, we may assume, for convenience, that $V_{1}$ and $V_{2}$ act on the same space $H$ and $F \equiv V_{1}-V_{2}$ has finite rank. Let $x_{1}, \ldots, x_{k}$ be vectors which span the range of $F$ and let $K$ be the (closed) subspace spanned by $V_{1}^{n} x_{m}, n \geq 0$ and $1 \leq m \leq k$. Then $K$ is also spanned by $V_{2}^{n} x_{m}$, $n \geq 0$ and $1 \leq m \leq k$, and, in particular, $K$ is invariant for $V_{1}$ and $V_{2}$ and hence for $F$. Thus we have the triangulations

$$
V_{1}=\left[\begin{array}{cc}
V_{11} & * \\
0 & V_{12}
\end{array}\right], \quad V_{2}=\left[\begin{array}{cc}
V_{21} & * \\
0 & V_{22}
\end{array}\right], \quad F=\left[\begin{array}{cc}
F_{1} & F_{2} \\
0 & 0
\end{array}\right]
$$

on $H=K \oplus K^{\perp}$. Let $U_{i j}$ be the singular unitary part of $V_{i j}, i, j=1,2$. It was shown in [8, Lemma 4.4] that $U_{1 s}=U_{11} \oplus U_{12}$ and $U_{2 s}=U_{21} \oplus U_{22}$. We have $\mu\left(U_{i 1}\right) \leq \mu\left(V_{i 1}\right)<\infty, i=1,2$, and $U_{12}=U_{22}$, the latter because $V_{12}=V_{22}$. The unitary equivalence of $U_{1 a}$ and $U_{2 a}$ follows from a result of Carey [3, Proposition], and the equality of $n_{1}$ and $n_{2}$ from the Fredholm index theory [4, Theorem XI.3.11]. This proves our necessity assertion.

To prove the sufficiency, assume that $V_{j}=U_{j} \oplus W, j=1,2$. Let $n=$ $n_{1}=n_{2} \geq 1$. Since $U_{j}$ is a singular unitary operator with finite multiplicity, by Lemma 2.4 there is a finite-rank operator $F_{j}$ such that $U_{j} \oplus S^{(n)}$ and $S^{(n)}+F_{j}$ are unitarily equivalent. Hence $U_{1} \oplus S^{(n)}$ is unitarily equivalent to a finite-rank perturbation of $U_{2} \oplus S^{(n)}$. This yields our assertion that $\operatorname{rank}\left(V_{1}-U^{*} V_{2} U\right)<\infty$ for some unitary $U$.

Related results on finite-rank perturbations of more general contractions may be found in $[7,8,2,1]$.

Acknowledgements. The second author wants to thank Hwa-Long Gau for some discussions on Theorem 2.1. The research of the first author was supported by NSERC; that of the second author by the National Science Council of the Republic of China under project NSC-93-2115-M-009-017. 


\section{References}

[1] C. Benhida, Unitary equivalence of operators and dilations, Studia Math. 164 (2004), 253-255.

[2] C. Benhida and D. Timotin, Finite rank perturbations of contractions, Integral Equations Operator Theory 36 (2000), 253-268.

[3] R. W. Carey, Trace class perturbations of isometries and unitary dilations, Proc. Amer. Math. Soc. 45 (1974), 229-234.

[4] J. B. Conway, A Course in Functional Analysis, 2nd ed., Springer, New York, 1990.

[5] P. R. Halmos, A Hilbert Space Problem Book, 2nd ed., Springer, New York, 1982.

[6] Y. Nakamura, One-dimensional perturbations of the shift, Integral Equations Operator Theory 17 (1993), 373-403.

[7] K. Takahashi and P. Y. Wu, Dilation to the unilateral shifts, ibid. 32 (1998), 101-113.

[8] P. Y. Wu and K. Takahashi, Singular unitary dilations, ibid. 33 (1999), 231-247.

Department of Mathematics

University of Toronto

Toronto, Ontario M5S 2E4, Canada

E-mail: choi@math.toronto.edu
Department of Applied Mathematics National Chiao Tung University Hsinchu 300, Taiwan E-mail: pywu@math.nctu.edu.tw 